\title{
Sedentary behavior, physical activity and renal function in middle- aged and older adults: isotemporal substitution modelling
}

\author{
Keisei Kosaki \\ Waseda Univeristy \\ Koichiro Tanahashi \\ Kyoto Pharmaceutical University \\ Masahiro Matsui \\ University of Tsukuba \\ Nobuhiko Akazawa \\ Japan Institute of Sports Sciences \\ Yosuke Osuka \\ Tokyo Metropolitan Institute of Gerontology \\ Kiyoji Tanaka \\ University of Tsukuba \\ David W. Dunstan \\ Baker Heart and Diabetes Institute \\ Neville Owen \\ Baker Heart and Diabetes Institute \\ Ai Shibata \\ University of Tsukuba \\ Koichiro Oka \\ Waseda University \\ Seiji Maeda ( $\nabla$ maeda.seiji.gn@u.tsukuba.ac.jp ) \\ University of Tsukuba https://orcid.org/0000-0003-1642-4644
}

\section{Research article}

Keywords: Sedentary time, Physical activity time, Estimated glomerular filtration rate, Chronic kidney disease, Isotemporal substitution modelling

Posted Date: January 7th, 2020

DOl: https://doi.org/10.21203/rs.2.20257/v1

License: (1) This work is licensed under a Creative Commons Attribution 4.0 International License. Read Full License

Version of Record: A version of this preprint was published at BMC Nephrology on June 3rd, 2020. See the published version at https://doi.org/10.1186/s12882-020-01869-8. 


\section{Abstract}

Background: Both physical inactivity and sedentary behavior (too much sitting) can contribute to renal dysfunction. However, the potential benefits of behavior change (e.g., replacing sedentary behavior with physical activity) on renal function are not fully understood. We used isotemporal substitution to model potential impacts on renal function of replacing time from one behavior to another in middle-aged and older adults. Methods: For 174 Japanese adults (aged 50 - 83 years; $76 \%$ women), the time spent in sedentary behavior, light-intensity physical activity (LPA), and moderate to vigorous-intensity physical activity (MVPA) were assessed using uniaxial accelerometers. Renal function was evaluated by the estimated glomerular filtration rate (eGFR) from serum creatinine and cystatin C levels. Results: In univariate analyses, eGFR was significantly, albeit weakly, correlated with time spent in sedentary behavior ( $r s=-0.229)$, LPA $(r s=0.265)$ and with MVPA ( $r$ s = 0.353). In the isotemporal substitution models, replacement of $30 \mathrm{~min} /$ day of sedentary behavior with equivalent LPA time was not significantly associated with eGFR ( $\beta=2.25, p=0.111)$; however, replacement with the same amount of MVPA was beneficially associated with eGFR $(\beta=5.51, p<0.05)$. Conclusions: These cross-sectional findings suggest that both sedentary behavior (detrimentally) and physical activity (beneficially) may be contributors to maintaining renal function and that replacing sedentary behavior with MVPA may benefit renal health in middle-aged and older adults.

\section{Background}

Chronic kidney disease (CKD), defined as persistent renal dysfunction or renal damage, is one of the major non-communicable diseases with a high prevalence worldwide [1]. For example, CKD in the Japanese adult population is estimated to affect about one in eight and has been increasing among those who are middle-aged and older [2]. As these trends of CKD prevalence are expected to continue, especially in countries with ageing populations, including Japan [3], there is the need to identify practical countermeasures for preventing the onset and progression of CKD in middle-aged and older adults.

Insufficient moderate- to vigorous-intensity physical activity (MVPA) is associated with the onset of renal dysfunction [4]. Subsequently, physical inactivity has been recognized recently as one of the important targets for intervention in renal care [5]. In addition, new evidence suggests that sedentary behavior defined as any waking behavior characterized by an energy expenditure $\leq 1.5$ metabolic equivalents, such as television viewing time [6] may be another risk factor for renal dysfunction [7, 8]. Taken together, these epidemiological findings suggest the importance of behavioral approaches for renal care and the possibility that specific behavior change (e.g., replacing sedentary behavior with physical activity) may contribute to preventing renal dysfunction. However, the potential impacts of replacing sedentary behavior with physical activity on renal function are not well-understood.

Isotemporal substitution modelling is a novel statistical approach that enables the estimation of associations when replacing time from one behavior to another, whilst keeping total time fixed and other behaviors fixed [9]. Recent cross-sectional studies using this approach have demonstrated that replacing time spent in sedentary behavior with equivalent physical activity can be associated beneficially with cardiometabolic biomarkers, health-related quality of life, and physical function [10-13].

We used isotemporal substitution modelling to examine cross-sectional associations of accelerometer-derived sedentary behavior and the different physical activity intensities (LPA and MVPA) with renal function in middle-aged and older adults.

\section{Methods}

\section{Participants}

This cross-sectional study used the data from 200 participants who were recruited for an interventional study via local newspaper advertisements between 2014 and 2015. In total, there were 180 eligible participants, after excluding those without objectively evaluated sedentary behavior and physical activity or those who had insufficient accelerometer data. After excluding participants who had missing values of required variables such as blood samples $(n=6)$, the final analyses were conducted 174 middle-aged and older Japanese adults (Fig. 1). This study was approved by the Ethics Committee in University of Tsukuba (Tai 019-19) and conformed to the principles outlined in the Declaration of Helsinki, and all participants provided written informed consent.

\section{Sedentary behavior and physical activity}

The time spent in sedentary behavior, light-intensity physical activity (LPA), and MVPA were assessed using a uniaxial accelerometer (Lifecorder, Suzuken Co., Ltd., Nagoya, Japan) that samples vertical acceleration signals in the range from 0.06 to $1.94 \mathrm{G}$ at $32 \mathrm{~Hz}$. The accuracy and detailed algorithm of this accelerometer has been described elsewhere [14]. The epoch length was 1 minute, the time used by the accelerometer for measuring activity. Participants were instructed to wear constantly the accelerometer on the level of participant's waist 
during waking and sleeping hour for 7 consecutive days, except while bathing and swimming. A day with at least 10 hours of wear time was considered valid. This accelerometer records the scores of physical activity intensity consisted of a scale from 0 - 9 (level 0 : rest; level 0.5 : micro activity; level 1 - 9: movement) according to the acceleration signal patterns [15]. In the present study, these scores were reclassified into four activity levels based on the previous investigation [16] as follows: sedentary or sleep ( $\leq 1.5$ Mets: level 0 - 0.5$)$, light (1.6 - 2.9 Mets: level $1-3)$, moderate (3.0 - 6.0 Mets: level $4-6)$, and vigorous (> 6.0 Mets: level $7-9)$, and were reported as the time spent in each activity level. The time spent in sedentary behavior was calculated as the time of sedentary or sleep (<1.5 Mets: level $0-0.5)$ minus the sleep time assessed by the validated questionnaire (The Japanese version of the Pittsburgh Sleep Quality Index). Also, the moderate and vigorous physical activity time were combined to form the time spent in MVPA.

\section{Renal function}

Estimated glomerular filtration rate (eGFR) was calculated by the Japanese eGFR equations based on standardized serum creatinine or cystatin C as follows: eGFR ${ }_{c r}\left(\mathrm{~mL} / \mathrm{min} / 1.73 \mathrm{~m}^{2}\right)=194 \times$ serum creatinine ${ }^{-1.094} \times$ Age $^{-0.287} \times 0.739$ (if female), eGFR ${ }_{\text {cys }}\left(\mathrm{mL} / \mathrm{min} / 1.73 \mathrm{~m}{ }^{2}\right)=$ $\left\{104 \times\right.$ serum cystatin $\mathrm{C}^{-1.019} \times 0.996{ }^{\text {Age }} \times 0.929$ (if female) $\}-8[17,18]$. To improve estimated accuracy, the average values of eGFR ${ }_{\text {cr }}$ and eGFR $_{\text {cys }}$ were used as the index of renal function.

\section{Covariates}

Brachial systolic and diastolic blood pressure and heart rate were simultaneously measured using semi-automatic vascular testing device with electrocardiogram and oscillometric extremities cuffs (form PWV/ABI, Colin Medical technology, Japan). Fasting blood samples were collected in the morning following a more than 12-hour overnight fasting to measure serum or plasma concentrations of high-density lipoprotein (HDL) cholesterol, low-density lipoprotein (LDL) cholesterol, triglyceride, glucose, creatinine, and cystatin C.

\section{Statistical analysis}

All statistical analyses were performed using SPSS Statistics 25.0 (IBM Corp., Tokyo, Japan). Data were presented as the means \pm SD (for normal distribution), median with interquartile range (for skewed distribution), or frequency counts (for categorical data), as appropriate. Univariate linear associations of sedentary behavior, LPA, and MVPA with eGFR were examined using Spearman's rank correlation coefficients $\left(r_{s}\right)$. Joint associations of sedentary behavior, LPA, and MVPA (four groups stratified according to each median value: higher sedentary behavior/higher physical activity, higher sedentary behavior/lower physical activity, lower sedentary behavior/higher physical activity, lower sedentary behavior/lower physical activity) with eGFR were examined using two-way analysis of co-variance (ANCOVA) to adjust for total waking time and covariates including age, sex, body mass index, systolic blood pressure, heart rate, HDL cholesterol, LDL cholesterol, triglycerides, fasting blood glucose, antihypertensive medicine, lipid-lowering medicine, hypoglycemic medicine, and current smoking. Independent associations of the exposure variables (sedentary behavior, LPA, and MVPA) with the outcome variable (eGFR) were assessed using three multiple linear regression models including a single factor model, partition model, and isotemporal substitution model. For enhanced interpretability of the results, the exposure variables (sedentary behavior, LPA, and MVPA) were scaled to 30 min/day units, respectively [13]. Briefly, the single factor models evaluated separately the associations between each exposure variable and eGFR, with adjustment for total waking time and covariates (Model $1-3$ ). The partition model evaluated simultaneously the associations between all exposure variables and eGFR, with adjustment for covariates, but without adjusting for total waking time (Model 4). The isotemporal substitution models estimated the substitution associations between replacing one exposure variable with an equal amount of time in another exposure variable (e.g., replacement of $30 \mathrm{~min} /$ day of sedentary behavior with $30 \mathrm{~min} /$ day of MVPA) and GFR average (Model 5 - 7). This estimation can be accomplished by omitting replacing target exposure variable from the model and entering total waking time and covariates. A more detailed description of these regression models is presented elsewhere [9].

\section{Results}

Characteristics of the participants are shown in Table 1. The mean age was $64 \pm 7$ years and most of the participants were women (76\%). On average, the majority of total waking time (1056 $\pm 61 \mathrm{~min} /$ day) was spent in sedentary behavior (970 $\pm 64 \mathrm{~min} /$ day), with $67 \pm 25 \mathrm{~min}$ of LPA and $19 \pm 14 \mathrm{~min}$ of MVPA per day. The mean values of eGFR were $85 \pm 14 \mathrm{~mL} / \mathrm{min} / 1.73 \mathrm{~m}^{2}$, and a small number of participants ( $\mathrm{n}=6$ ) were less than $60 \mathrm{~mL} / \mathrm{min} / 1.73 \mathrm{~m}^{2}$. Several participants were taking medications, including an antihypertensive agent (20\%), lipid-lowering agent (10\%), and/or hypoglycemic agent (1\%). 
Table 1

Characteristics of selected participants $(n=174)$

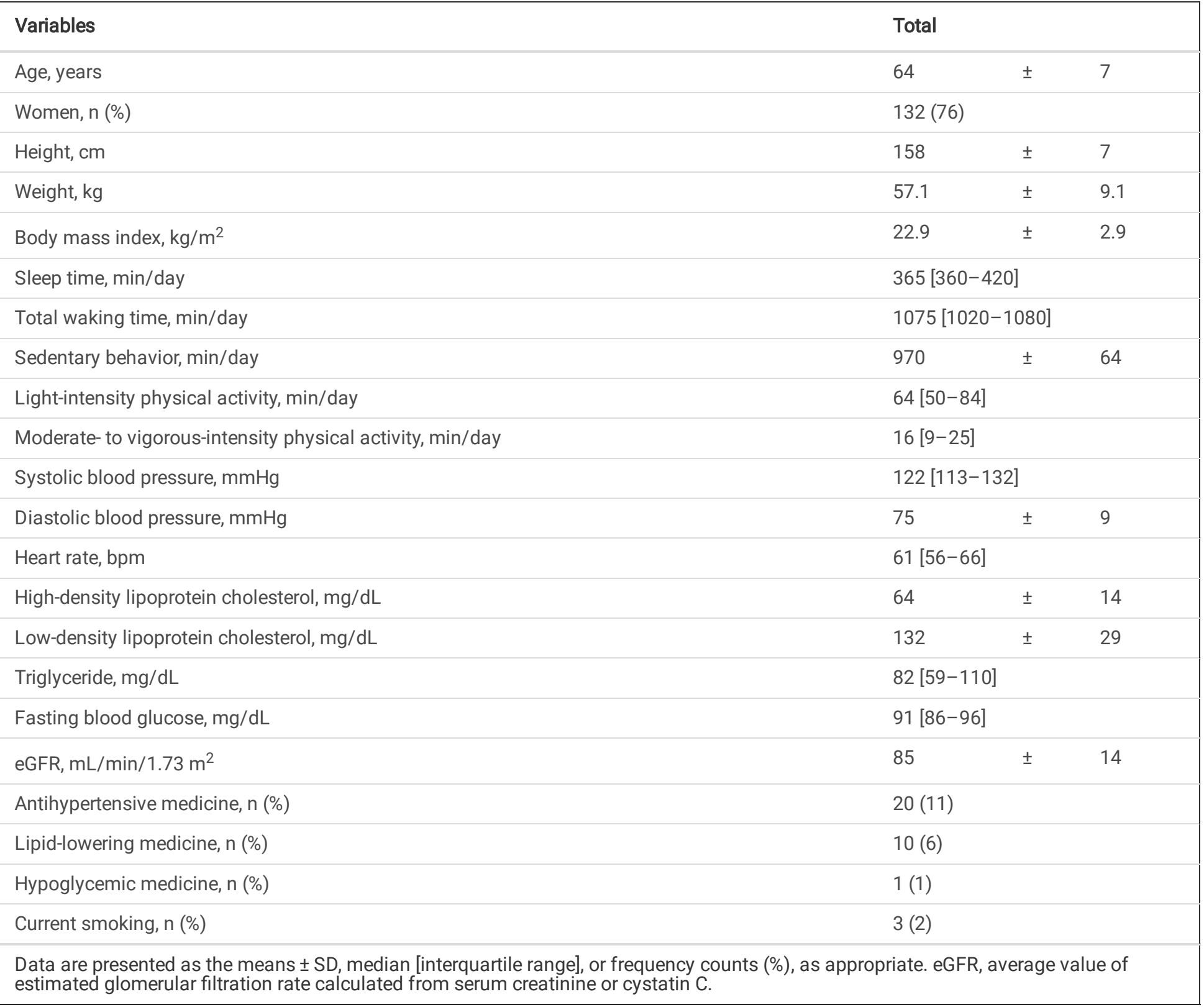

The results of univariate linear analyses are presented in Fig. 2. The time spent in sedentary behavior was significantly and negatively, albeit weakly, correlated with eGFR $\left(r_{s}=-0.229, p<0.05\right)$. The time spent in LPA and MVPA were significantly and positively correlated with eGFR $\left(r_{s}=0.265, p<0.001, r_{s}=0.353, p<0.001\right.$, respectively).

Figure 3 shows the joint associations of sedentary behavior, LPA and MVPA (four groups stratified according to each median value) with eGFR. Although there was no statistically significant interaction between sedentary behavior and LPA $(p=0.184)$ or MVPA $(p=0.834)$ in their association with eGFR, the mean values of eGFR were lowest in those with higher sedentary behavior and lower LPA $(81.1$ [95\% Cl: 76.9, 85.3] $\left.\mathrm{mL} / \mathrm{min} / 1.73 \mathrm{~m}^{2}\right)$ or MVPA $\left(79.3[95 \% \mathrm{Cl}: 74.9,83.7] \mathrm{mL} / \mathrm{min} / 1.73 \mathrm{~m}^{2}\right)$, respectively.

The results from three multiple linear regression models with adjusting for covariates are summarized in Table 2 . The single factor models (Model 1-3) showed that sedentary behavior was significantly and negatively associated with eGFR $(\beta=-3.31, p<0.001)$, conversely, both LPA and MVPA were significantly and positively associated with eGFR $(\beta=3.61, p<0.05, \beta=7.07, p<0.05$, respectively). The partition model (Model 4) showed that only MVPA was significantly associated with eGFR $(\beta=4.71, p<0.05)$. The isotemporal substitution models (Model $5-7$ ) showed that replacement of $30 \mathrm{~min} /$ day of sedentary behavior with equivalent LPA time was not significantly associated with eGFR ( $\beta$ $=2.25, p=0.111)$, however, replacement with the same amount of MVPA was modestly but significantly associated with the eGFR $(\beta=5.51, p$ $<0.05)$. Replacement of $30 \mathrm{~min} /$ day of LPA with equivalent MVPA time was not significantly associated with eGFR $(\beta=3.25, p=0.312)$. 
Table 2

Single factor, partition, and isotemporal substitution models for renal function evaluated by eGFR

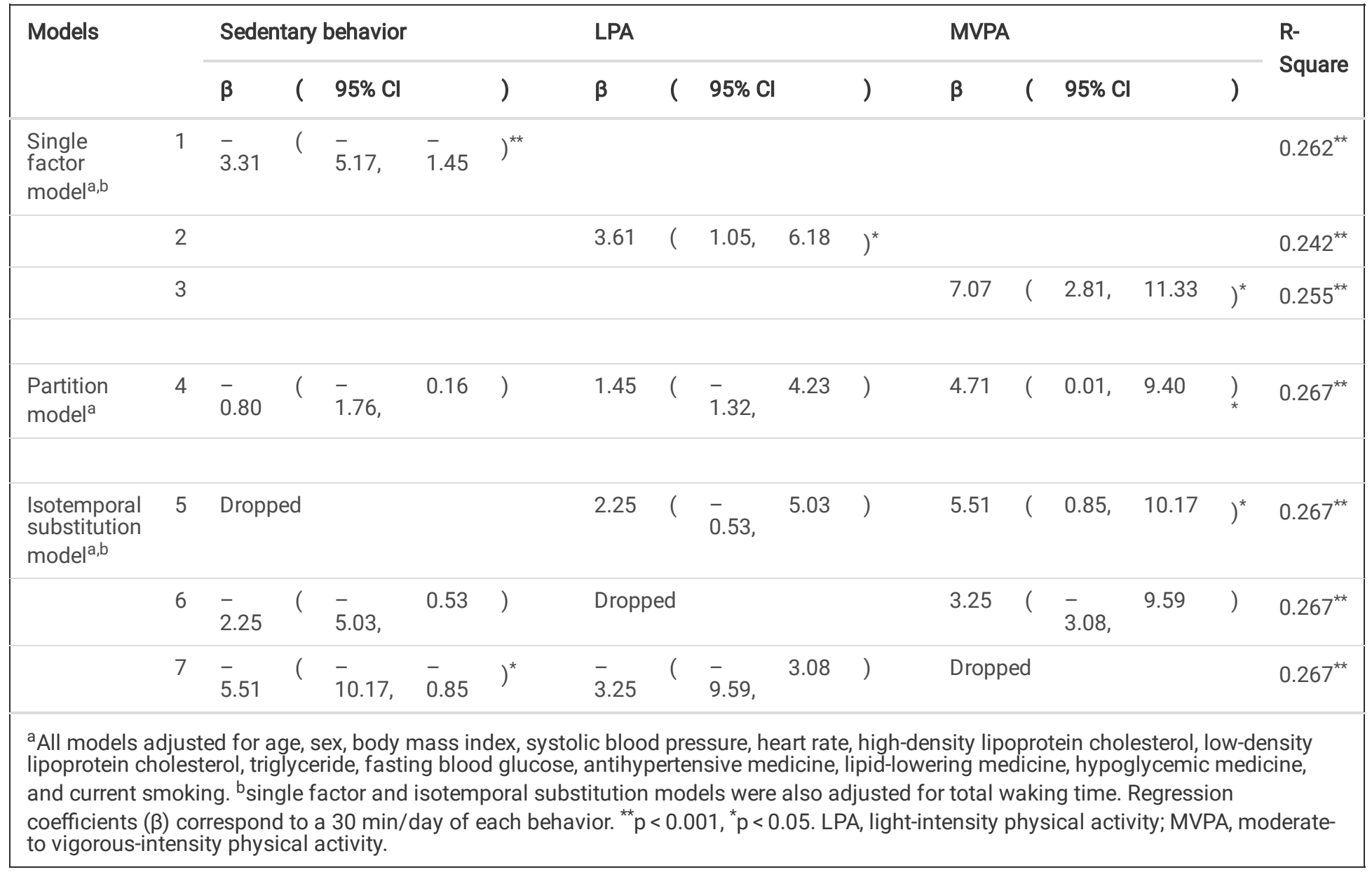

\section{Discussion}

In this cross-sectional study of middle-aged and older adults, we examined the associations of accelerometer-derived sedentary behavior and physical activity with renal function and potential renal impacts of replacing sedentary behavior with the different intensities of physical activity. Time spent in sedentary behavior, LPA, and MVPA were significantly associated with eGFR. Furthermore, isotemporal substitution modelling showed that replacement of $30 \mathrm{~min} /$ day of sedentary behavior with equivalent MVPA time was significantly associated with the better eGFR. These findings suggest that both sedentary behavior (detrimentally) and physical activity (beneficially) may be contributing factors to maintaining renal function, and replacing sedentary behavior with physical activity (MVPA) may benefit renal health in middle-aged and older adults.

Several previous studies have reported the associations of sedentary behavior and physical activity with renal function [7, 8, 19-25]. However, only a few studies have used accelerometer-derived measures of sedentary behavior and physical activity [19, 20, 23], most studies have relied on self-reported measures derived from questionnaires which may be limited in quantifying the degree of behavior. In addition, there is no study examining the potential impacts of replacing sedentary behavior with physical activity on renal function using the isotemporal substitution modelling. To our knowledge, this is the first study to examine the associations of accelerometer-derived sedentary behavior and physical activity with renal function using the isotemporal substitution modelling in Japanese adults who have been reported to have the highest sitting times (medians $\geq 360 \mathrm{~min} /$ day) [26].

This study has also shown the mean values of eGFR were lowest in those with higher sedentary behavior and lower physical activity (LPA and MVPA). However, there was no statistically significant interaction between sedentary behavior and physical activity with respect to the association with the eGFR. This is because the influences of physical activity, especially MVPA on eGFR might be apparently stronger than those of sedentary behavior. Univariate analyses also showed that the associations between MVPA and eGFR ( $r_{s}=0.353$ ) were stronger than those of sedentary behavior $\left(r_{s}=\unrhd 0.229\right)$. Therefore, the combination of higher sedentary behavior and lower physical activity are likely to be strongest with respect to having a lower renal function; however, the MVPA emerged as potentially having a more significant influence on renal function than did sedentary behavior. 
Beneficial effects of regularly performed physical activity (i.e., exercise training) on several medical conditions have been demonstrated in the general population and in patients with CKD. The Kidney Disease: Improving Global Outcomes (KDIGO) guideline mainly intends to maintain and improve cardiovascular health and tolerance and recommends that patients with CKD should undertake moderate physical activity for at least $30 \mathrm{~min}$ five times per week in line with recommendations for the general population [27]. Furthermore, recent metaanalyses have also shown the possibility that exercise training slightly improves eGFR in patients with CKD [28, 29]. However, the beneficial effects of exercise training on renal function are not likely to be conclusive and remain controversial due to a limited small number of studies in both meta-analyses. We estimated the potential impacts of specific behavior change on renal function using isotemporal substitution modelling and revealed that replacement of $30 \mathrm{~min} /$ day of sedentary behavior with equivalent physical activity (MVPA) was significantly associated with the better eGFR $\left(+5.51[95 \% \mathrm{Cl}: 0.85,10.17] \mathrm{mL} / \mathrm{min} / 1.73 \mathrm{~m}^{2}\right)$. This finding supports and expands on the results of several previous interventional studies and the KDIGO recommendation of physical activity.

The strengths of this study are the use of accelerometers to objectively assess each behavior, and the clinically interpretable results obtained from the isotemporal substitution modelling. However, there are several limitations. First, the nature of cross-sectional investigation limits our ability to judge the precise causal links between sedentary behavior, physical activity, and renal function. We cannot deny the possibility that renal function may be contributing factors to maintaining sedentary behavior and physical activity. Second, the potential renal impacts examined using the isotemporal substitution models were estimations, and the actual impacts of specific behavior change on renal function remains unknown. Third, we evaluated only an eGFR as renal function in this study; however, other comprehensive evaluations by several urinary biomarkers such as urinary albumin will be needed. Fourth, a generalization of our findings may be difficult due to the relatively small sample size, a single-center study, and the selective populations (i.e., middle-aged and older Japanese adults). Finally, the reasons for the better renal function when replacing sedentary behavior with physical activity remains equivocal. Considering the findings of previous basic research [30], several mediating factors including anti-oxidant defense and anti-inflammatory environment may be involved in the mechanism underlying the renal benefits of specific behavior change. Further interventional studies, such as randomized controlled trials, examining the effects of moderate- to vigorous-intensity exercise on renal health are needed to address these limitations and provide a more definitive interpretation of the present findings.

\section{Conclusion}

Sedentary behavior (detrimentally) and physical activity (LPA and MVPA) (beneficially) were significantly associated with renal function evaluated by eGFR. Findings from isotemporal substitution modelling suggest that replacement of $30 \mathrm{~min} /$ day of sedentary behavior with equivalent MVPA time may contribute to the better renal function in middle-aged and older adults. These findings provide important preliminary evidence on the potential renal benefits of decreasing sedentary time and increasing physical activity time and include important public health implications.

\section{Abbreviations}

LPA: light-intensity physical activity; MVPA: moderate to vigorous-intensity physical activity; eGFR: estimated glomerular filtration rate; CKD: chronic kidney disease; HDL: high-density lipoprotein; LDL: low-density lipoprotein; KDIGO: Kidney Disease: Improving Global Outcomes

\section{Declarations}

\section{Ethics approval and consent to participate}

This study was approved by the Ethics Committee in University of Tsukuba (Tai 019-19) and conformed to the principles outlined in the Declaration of Helsinki, and all participants provided written informed consent.

\section{Consent for publication}

Not applicable.

\section{Availability of data and materials}

The datasets generated and/or analysed during the current study are not publicly available due to the ongoing nature of this study but are available from the corresponding author on reasonable request.

\section{Competing interests}

The authors declare that they have no competing interests. 


\section{Funding}

This work was supported in part a Grant-in-Aid for Scientific Research KAKENHI from the Ministry of Education, Culture, Sports, Science, and Technology, Japan (19H03995). K.K. was recipients of a Grant-in-Aid for Research Fellowships of Japan Society for the Promotion of Science for Young Scientists (19J01099).

\section{Authors' contributions}

K.K., A.S., K.O., and S.M.: research conception; K.K., K.Tanahashi., M.M., N.A., and Y.O: data acquisition/analysis; K.K., K.Tanaka., D.D., N.O., A.S., K.O., and S.M.: data interpretation; K.K.: statistical analysis; D.D., N.O., K.O., and S.M: supervision or mentorship.

\section{Acknowledgements}

We thank our (S.M.'s) laboratory members in the University of Tsukuba for their technical assistance.

\section{References}

1. Xie Y, Bowe B, Mokdad AH, Xian H, Yan Y, Li T, Maddukuri G, Tsai CY, Floyd T, Al-Aly Z: Analysis of the Global Burden of Disease study highlights the global, regional, and national trends of chronic kidney disease epidemiology from 1990 to 2016 . Kidney Int 2018 , 94(3):567-581.

2. Imai E, Horio M, Iseki K, Yamagata K, Watanabe T, Hara S, Ura N, Kiyohara Y, Hirakata H, Moriyama T et al: Prevalence of chronic kidney disease (CKD) in the Japanese general population predicted by the MDRD equation modified by a Japanese coefficient. Clin Exp Nephrol 2007, 11(2):156-163.

3. Jha V, Garcia-Garcia G, Iseki K, Li Z, Naicker S, Plattner B, Saran R, Wang A, Yang C: Chronic kidney disease: global dimension and perspectives. Lancet 2013, 382(9888):260-272.

4. Hallan S, de Mutsert R, Carlsen S, Dekker FW, Aasarod K, Holmen J: Obesity, smoking, and physical inactivity as risk factors for CKD: are men more vulnerable? Am J Kidney Dis 2006, 47(3):396-405.

5. Zelle DM, Klaassen G, van Adrichem E, Bakker SJ, Corpeleijn E, Navis G: Physical inactivity: a risk factor and target for intervention in renal care. Nat Rev Nephrol 2017, 13(3):152-168.

6. Tremblay MS, Aubert S, Barnes JD, Saunders TJ, Carson V, Latimer-Cheung AE, Chastin SFM, Altenburg TM, Chinapaw MJM, Participants STCP: Sedentary Behavior Research Network (SBRN) - Terminology Consensus Project process and outcome. Int J Behav Nutr Phys Act 2017, 14(1):75.

7. Hawkins M, Newman AB, Madero M, Patel KV, Shlipak MG, Cooper J, Johansen KL, Navaneethan SD, Shorr RI, Simonsick EM et al: TV Watching, but Not Physical Activity, Is Associated With Change in Kidney Function in Older Adults. J Phys Act Health 2015, 12(4):561568 .

8. Lynch BM, White SL, Owen N, Healy GN, Chadban SJ, Atkins RC, Dunstan DW: Television viewing time and risk of chronic kidney disease in adults: the AusDiab Study. Ann Behav Med 2010, 40(3):265-274.

9. Mekary RA, Willett WC, Hu FB, Ding EL: Isotemporal substitution paradigm for physical activity epidemiology and weight change. Am J Epidemiol 2009, 170(4):519-527.

10. Yasunaga A, Shibata A, Ishii K, Inoue S, Sugiyama T, Owen N, Oka K: Replacing sedentary time with physical activity: effects on healthrelated quality of life in older Japanese adults. Health Qual Life Outcomes 2018, 16(1):240.

11. Yasunaga A, Shibata A, Ishii K, Koohsari MJ, Inoue S, Sugiyama T, Owen N, Oka K: Associations of sedentary behavior and physical activity with older adults' physical function: an isotemporal substitution approach. BMC Geriatr 2017, 17(1):280.

12. Hamer M, Stamatakis E, Steptoe A: Effects of substituting sedentary time with physical activity on metabolic risk. Med Sci Sports Exerc 2014, 46(10):1946-1950.

13. Buman MP, Winkler EA, Kurka JM, Hekler EB, Baldwin CM, Owen N, Ainsworth BE, Healy GN, Gardiner PA: Reallocating time to sleep, sedentary behaviors, or active behaviors: associations with cardiovascular disease risk biomarkers, NHANES 2005-2006. Am J Epidemiol 2014, 179(3):323-334.

14. Kumahara H, Schutz Y, Ayabe M, Yoshioka M, Yoshitake Y, Shindo M, Ishii K, Tanaka H: The use of uniaxial accelerometry for the assessment of physical-activity-related energy expenditure: a validation study against whole-body indirect calorimetry. Br $\mathrm{J}$ Nutr 2004 , 91(2):235-243. 
15. Hikihara Y, Tanaka S, Ohkawara K, Ishikawa-Takata K, Tabata I: Validation and comparison of 3 accelerometers for measuring physical activity intensity during nonlocomotive activities and locomotive movements. J Phys Act Health 2012, 9(7):935-943.

16. Ishii K, Shibata A, Adachi M, Mano Y, Oka K: Objectively Measured Sedentary Behavior, Obesity, and Psychological Well-Being: A CrossSectional Study of Japanese Schoolchildren. J Phys Act Health 2017, 14(4):270-274.

17. Horio M, Imai E, Yasuda Y, Watanabe T, Matsuo S, Collaborators Developing the Japanese Equation for Estimated GFR: GFR estimation using standardized serum cystatin C in Japan. Am J Kidney Dis 2013, 61(2):197-203.

18. Matsuo S, Imai E, Horio M, Yasuda Y, Tomita K, Nitta K, Yamagata K, Tomino Y, Yokoyama H, Hishida A et al: Revised equations for estimated GFR from serum creatinine in Japan. Am J Kidney Dis 2009, 53(6):982-992.

19. Martens RJH, van der Berg JD, Stehouwer CDA, Henry RMA, Bosma H, Dagnelie PC, van Dongen M, Eussen S, Schram MT, Sep SJS et al: Amount and pattern of physical activity and sedentary behavior are associated with kidney function and kidney damage: The Maastricht Study. PLoS One 2018, 13(4):e0195306.

20. Glavinovic T, Ferguson T, Komenda P, Rigatto C, Duhamel TA, Tangri N, Bohm C: CKD and Sedentary Time: Results From the Canadian Health Measures Survey. Am J Kidney Dis 2018, 72(4):529-537.

21. Robinson-Cohen C, Littman AJ, Duncan GE, Weiss NS, Sachs MC, Ruzinski J, Kundzins J, Rock D, de Boer IH, Ikizler TA et al: Physical activity and change in estimated GFR among persons with CKD. J Am Soc Nephrol 2014, 25(2):399-406.

22. Bharakhada N, Yates T, Davies MJ, Wilmot EG, Edwardson C, Henson J, Webb D, Khunti K: Association of sitting time and physical activity with CKD: a cross-sectional study in family practices. Am J Kidney Dis 2012, 60(4):583-590.

23. Hawkins MS, Sevick MA, Richardson CR, Fried LF, Arena VC, Kriska AM: Association between physical activity and kidney function: National Health and Nutrition Examination Survey. Med Sci Sports Exerc 2011, 43(8):1457-1464.

24. Robinson-Cohen C, Katz R, Mozaffarian D, Dalrymple LS, de Boer I, Sarnak M, Shlipak M, Siscovick D, Kestenbaum B: Physical activity and rapid decline in kidney function among older adults. Arch Intern Med 2009, 169(22):2116-2123.

25. Finkelstein J, Joshi A, Hise MK: Association of physical activity and renal function in subjects with and without metabolic syndrome: a review of the Third National Health and Nutrition Examination Survey (NHANES III). Am J Kidney Dis 2006, 48(3):372-382.

26. Bauman A, Ainsworth BE, Sallis JF, Hagstromer M, Craig CL, Bull FC, Pratt M, Venugopal K, Chau J, Sjostrom M et al: The descriptive epidemiology of sitting. A 20-country comparison using the International Physical Activity Questionnaire (IPAQ). Am J Prev Med 2011, 41(2):228-235.

27. Levin A, Stevens PE: Summary of KDIGO 2012 CKD Guideline: behind the scenes, need for guidance, and a framework for moving forward. Kidney Int 2014, 85(1):49-61.

28. Yamagata K, Hoshino J, Sugiyama H, Hanafusa N, Shibagaki Y, Komatsu Y, Konta T, Fujii N, Kanda E, Sofue T et al: Clinical practice guideline for renal rehabilitation: systematic reviews and recommendations of exercise therapies in patients with kidney diseases. Renal Replacement Therapy 2019, 5(1).

29. Vanden Wyngaert K, Van Craenenbroeck AH, Van Biesen W, Dhondt A, Tanghe A, Van Ginckel A, Celie B, Calders P: The effects of aerobic exercise on eGFR, blood pressure and VO2peak in patients with chronic kidney disease stages 3-4: A systematic review and metaanalysis. PLoS One 2018, 13(9):e0203662.

30. Asghar M, George L, Lokhandwala MF: Exercise decreases oxidative stress and inflammation and restores renal dopamine D1 receptor function in old rats. Am J Physiol Renal Physiol 2007, 293(3):F914-919.

\section{Figures}




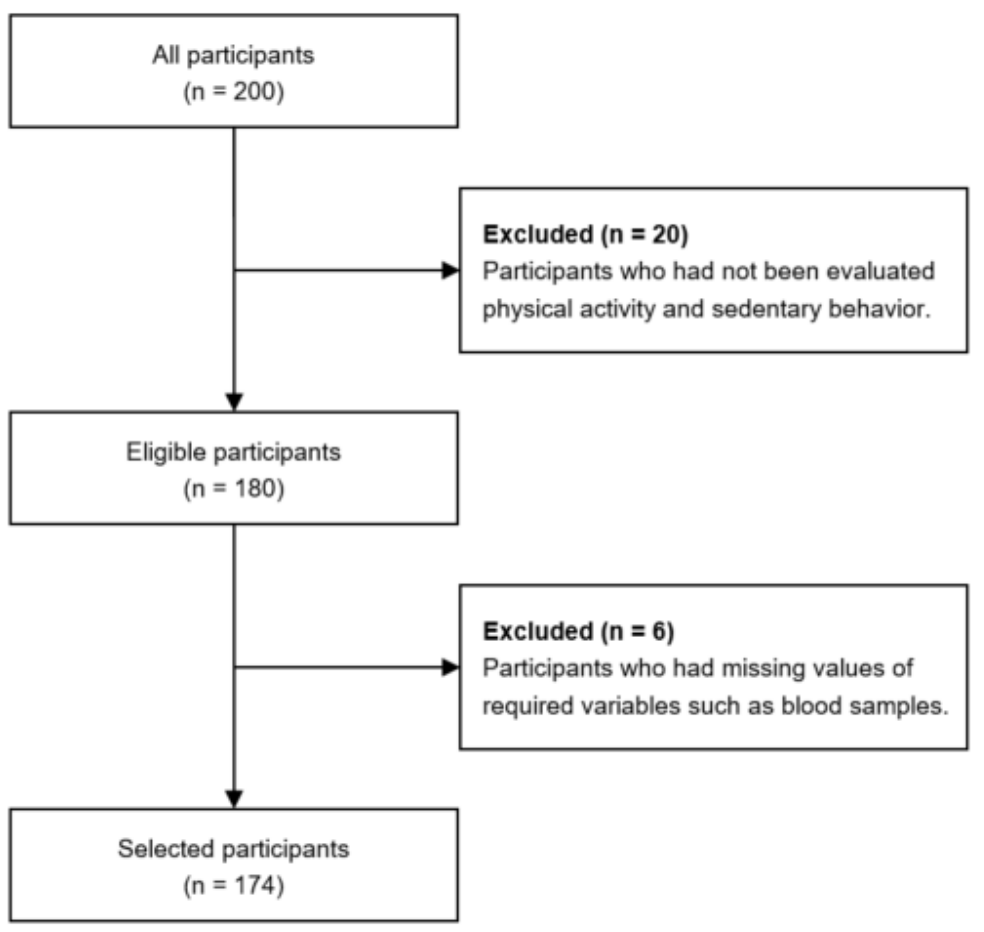

Figure 1

Flow diagram.
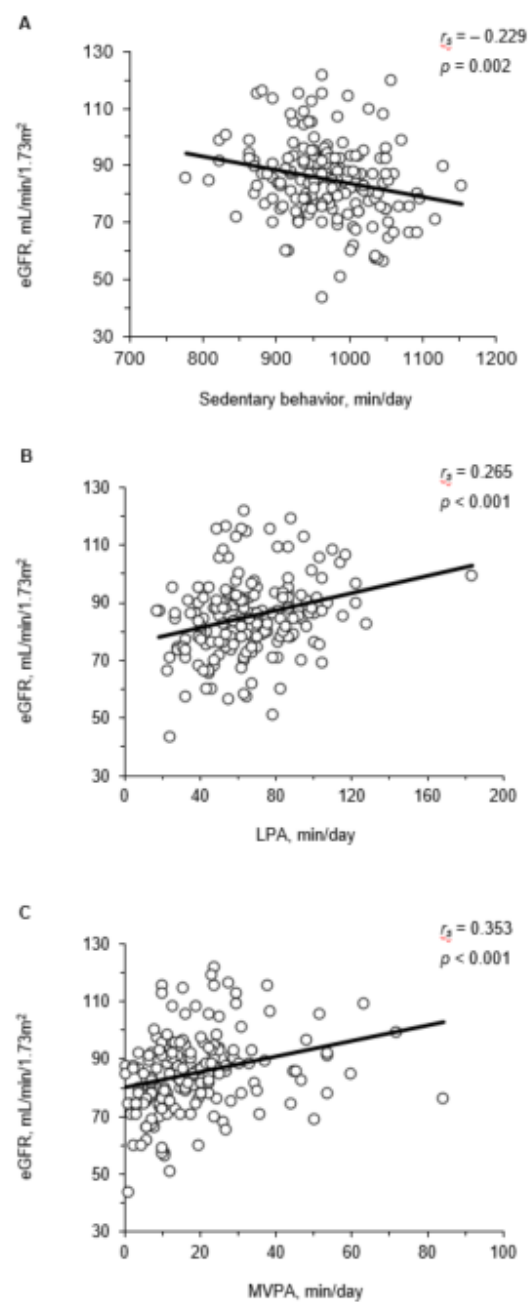
Figure 2

Univariate associations between sedentary behavior (A), light-intensity physical activity (LPA) (B), moderate-to vigorous-intensity physical activity (MVPA) (C) and estimated glomerular filtration rate (eGFR).
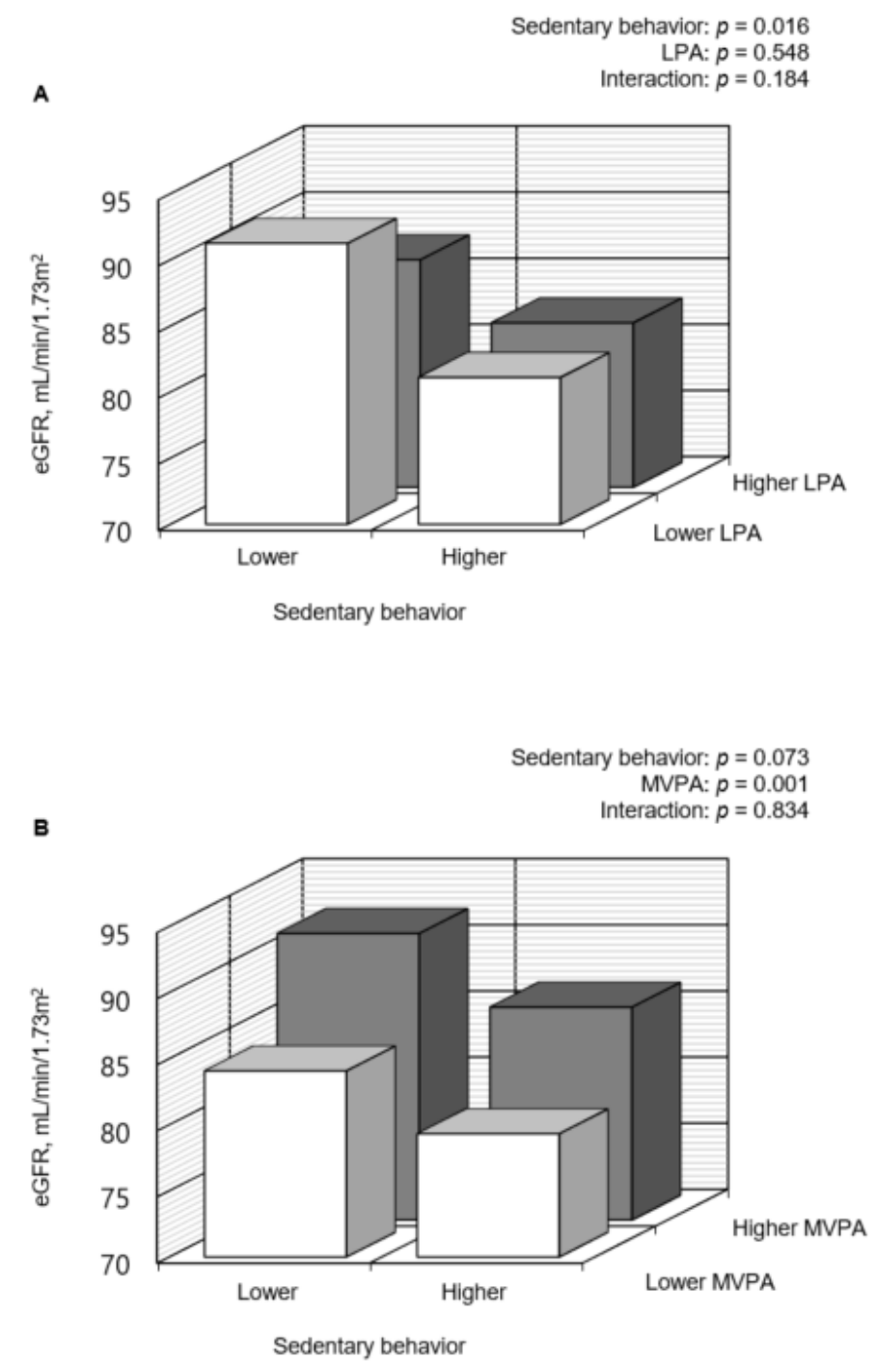

\section{Figure 3}

Associations of joint categories (four groups stratified according to each median value) of sedentary behavior, light-intensity physical activity (LPA) (A) and moderate-to vigorous-intensity physical activity (MVPA) (B) with estimated glomerular filtration rate (eGFR). p-values were evaluated using two-way ANCOVA after adjusted for age, sex, body mass index, systolic blood pressure, heart rate, HDL cholesterol, LDL cholesterol, triglyceride, fasting blood glucose, antihypertensive medicine, lipid-lowering medicine, hypoglycemic medicine, current smoking, and total waking time. 